\title{
QUASI-CLOSED PRIMARY COMPONENTS IN ABELIAN GROUP RINGS
}

\author{
PETER DANCHEV
}

\section{Introduction}

All groups considered in this work are assumed to be multiplicatively written abelian groups and all rings are commutative with identity of prime characteristic $p$ for some fixed prime $p$. We follow essentially throughout the notation and terminology to the abelian group theory of the excellent classical monographs of L. Fuchs [8]. All topological references are to the $p$-adic topology.

For $G$ a group and $R$ a ring, $R G$ will denote a group ring with a normed Sylow $p$-subgroup $S(R G)$, which is in the focus of our interest. The notations and terminology from the commutative group algebras theory of the nice book of $G$. Karpilovsky [10] will be followed.

This paper is a supplement and a generalization to our previous articles $[6,7]$. The main purpose that motivates the present research is the global investigation of the quasicompleteness of $S(R G)$ for large $R$ and $G$ on their minimal restrictions. In particular, as corollaries to our main results, we will obtain well-documented facts in [7] and other given by us in [6].

Before proving the central theorems, we need in the sequel some assertions stated in the following paragraph.

\section{Preliminary Statements}

First and foremost we start with some group-theoretic multiplicities very needed for our good presentation.

Proposition [6,7]. A nice subgroup of a closed p-group is closed. A balanced subgroup of a quasi-closed $p$-group is quasi-closed.

The next statement is valuable and due to Hill-Megibben (see also [8]).

Proposition [9]. Suppose $A$ is quasi-closed primary and $H$ is unbounded pure in $A$ such that $A / H$ is reduced (in particular $H$ can be choosen unbounded balanced in $A$ ). Then $A / H$ is closed.

Remark. We note that of some interest and importance is also the following more weak observation. If $A$ is quasi-closed $p$-primary and $H$ is balanced in $A$, then $A / H$

Received April 22, 2002. 
is quasi-closed. Really, let $B / H$ be pure in $A / H$. So [8] $B$ is pure in $A$. Therefore $(A / B)^{p^{w}} \cong(A / H / B / H)^{p^{w}}$ is divisible. This verifies that $A / H$ is quasi-closed since by a hypothesis it is clearly reduced.

On the other hand it is well to know that there exists a more simple proof of a weak variant (a special case) of Theorem 4.12 in [1]. It states as follows: Suppose $A$ is a $p$-group. Then $A$ is quasi-closed if and only if $A^{p^{n}}$ is quasi-closed for any natural $n$.

And so, if $A$ is $p$-primary and $A^{p^{n}}$ is quasi-closed for some fixed $n \in \mathbb{N}$, then so is $A$. In fact, $A^{p^{n}}$ is reduced whence the same is $A$. Let now $C$ be pure in $A$. Therefore $C^{p^{n}}$ is pure in $A^{p^{n}}$ and thus $\left(A^{p^{n}} / C^{p^{n}}\right)^{p^{w}}$ is divisible. But $A^{p^{n}} / C^{p^{n}} \cong(A / C)^{p^{n}}$ and consequently $\left(A^{p^{n}} / C^{p^{n}}\right)^{p^{w}} \cong(A / C)^{p^{w}}$, which completes the proof of the first half.

Conversely, take $A$ to be quasi-closed and $P$ to be a pure subgroup of $A^{p^{n}}$. Then invoking [2, Theorem 2.2] we observe that there is $C$ pure in $A$ with the property $C^{p^{n}}=P$. Therefore as above $\left(A^{p^{n}} / P\right)^{p^{w}}=\left(A^{p^{n}} / C^{p^{n}}\right)^{p^{w}} \cong(A / C)^{p^{w}}$ must be divisible, finishing the proof in general after all.

Recall that as usual $G_{p}$ is the $p$-torsion component of $G$. The following is well-known and documented, but is included here for a convenience.

Lemma $\left[\mathbf{5 , 6 , 7 ]}\right.$. For each ordinal $\varepsilon$ is valid that $S^{p^{\varepsilon}}(R G)=S\left(R^{p^{\varepsilon}} G^{p^{\varepsilon}}\right)$.

Now we are in position to attack.

Main Lemma. $G_{p}$ is balanced in $S(R G)$.

Proof. "nice property". Following [8] (by P. Hill), the subgroup $N$ of an abelian $p$-group $A$ is said to be nice if $(A / N)^{p^{\varepsilon}}=\left(A^{p^{\varepsilon}} N\right) / N$ for every ordinal $\varepsilon$. It is easily seen that the last is equivalent to $\cap_{\alpha<\varepsilon}\left(A^{p^{\alpha}} N\right)=A^{p^{\varepsilon}} N$ for each limit $\varepsilon$. Using this modification and the above lemma, to prove our claim it is enough to show only that $\cap_{\alpha<\varepsilon}\left(G_{p} S\left(R^{p^{\alpha}} G^{p^{\alpha}}\right)\right)=G_{p} S\left(R^{p^{\varepsilon}} G^{p^{\varepsilon}}\right)$. Indeed, given $x$ in the left handside. Hence $x=g_{p} \sum_{g \in G^{p^{\alpha}}} r_{g} g=g_{p}^{\prime} \sum_{g^{\prime} \in G^{p^{\beta}}} r_{g^{\prime}} g^{\prime}=\cdots$, where $g_{p}, g_{p}^{\prime} \in G_{p} ; r_{g} \in$ $R^{p^{\alpha}}, r_{g^{\prime}} \in R^{p^{\beta}}$ and $\beta$ is arbitrary with $\alpha<\beta \leq \varepsilon$. Furthermore $r_{g}=r_{g^{\prime}}$ and $g_{p} g=g_{p}^{\prime} g^{\prime}$. Since $\sum_{g \in G^{p^{\alpha}}} r_{g} g \in S(R G)$, then there is some $\bar{g} \in G_{p}^{p^{\alpha}}$. That is why $x=g_{p} \bar{g} \sum_{g \in G^{p^{\alpha}}} r_{g} g \bar{g}^{-1} \in G_{p} S\left(R^{p^{\varepsilon}} G^{p^{\varepsilon}}\right)$, because $g_{p} \bar{g} \in G_{p}$ and $\sum_{g \in G^{p^{\alpha}}} r_{g} g \bar{g}^{-1} \in$ $S(R G) \cap R^{p^{\beta}} G^{p^{\beta}}=S\left(R^{p^{\beta}} G^{p^{\beta}}\right)$, which verifies the first claim on niceness.

"isotype property". Employing the above lemma, for every ordinal number $\varepsilon$ we establish $G_{p} \cap S^{p^{\varepsilon}}(R G)=G_{p} \cap S\left(R^{p^{\varepsilon}} G^{p^{\varepsilon}}\right)=G_{p}^{p^{\varepsilon}}$, which verifies that $G_{p}$ is isotype in $S(R G)$. Combining the above two properties, we finish the proof.

Remark. The last two lemmas are proved also by W. May (see, for example, [10]) but when $R$ is a field. More precisely, May has shown that $G_{p}$ is balanced in $1+I\left(R G ; G_{p}\right)$. But (cf. $[5,6]) S(R G)=1+I\left(R G ; G_{p}\right)$ when $R$ is a field and so more precise $G_{p}$ is balanced in $S(R G)$, of course, for $R$ a field. The used our technique is different to that of May (see the cited book [10]).

We continue with (cf. [6])

Proposition. $S(R G)$ is reduced $\Leftrightarrow$ the maximal perfect subring of $R$ has nilpotents and $G$ is p-reduced or the maximal perfect subring has no nilpotents and $G_{p}$ is reduced. 
Moreover $S(R G)$ is separable $\Leftrightarrow R^{p^{w}}$ has nilpotents and $G$ is p-separable or $R^{p^{w}}$ is without nilpotents and $G_{p}$ is separable.

Proof. Follows by a direct application of the first lemma plus [5, Lemma 3]. The proof is completed.

Begin with the following paragraph in which are stated our major theorems selected in the next three sections, namely

\section{Main Results}

1. Quasi-closed $S(R G)$ for $p$-primary $G$

The main statement in this section, however, is the following (announced in [4] as Remark 12)

Theorem 1. Assume $G$ is a p-group. Then $S(R G)$ is quasi-closed if and only if $G$ is bounded.

Proof. If $G$ is bounded, then apparently (for example cf. [4]) $S(R G)$ is one also, whence it is quasi-closed [8].

Conversely, let now $S(R G)$ be quasi-closed and $G$ be unbounded. Therefore owing to the Main Lemma and to the proposition of Hill-Megibben, $S(R G) / G$ is closed. We claim then that $G$ is bounded. If not, then $G$ contains an unbounded basic subgroup $B=\coprod_{n=1}^{\infty} B_{n}$, where $B_{n}$ are homogeneous of order $p^{n}$. We set now the sequences $\varphi_{n}=$ $\prod_{i=1}^{n}\left(1+b_{i}^{p^{i-1}}-b_{i+1}^{p^{i}}\right)$ and $\Phi_{n}=\varphi_{n} G$, where $b_{n} \in B_{n}(n \in \mathbb{N})$ with $b_{n}^{p^{n-1}} \neq 1$. Evidently $\varphi_{n}^{p}=1$ whence $\left(\Phi_{n}\right)_{n=1}^{\infty} \in S(R G) / G$ is bounded at $p$, and moreover $\left(\Phi_{n}\right)_{n=1}^{\infty}$ is a Cauchy sequence since clearly $\left(\varphi_{n}\right)_{n=1}^{\infty}$ is. Next we shall show that $\Phi_{n}$ is not convergent (i.e. is divergent) in $S(R G) / G$. Otherwise $\Phi_{n} \rightarrow \Phi$ when $n \rightarrow \infty$ and where $\Phi \in S(R G) / G$ is the boundary of $\Phi_{n}, n \in \mathbb{N}$. Therefore by a definition, $\Phi \in \Phi_{n} S\left(R^{p^{k}} G^{p^{k}}\right) G / G$ for each $k \geq 2$ and $n \geq k$. That is why $\Phi=\left(\sum_{1}^{t} r_{i} g_{i}\right) G$ for some fixed $t \in \mathbb{N}$ and

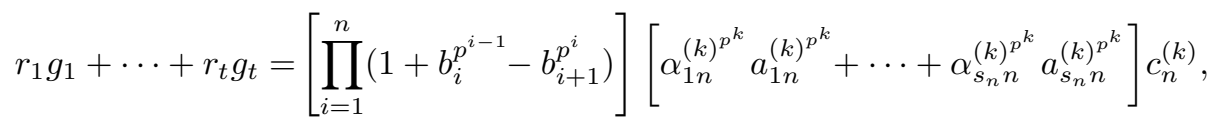

where $0 \neq r_{i} \in R, g_{i} \in G(1 \leq i \leq t), \sum_{1}^{t} r_{i}=1 ; \alpha_{1 n}^{(k)}, \ldots, \alpha_{s_{n} n}^{(k)} \in R, a_{1 n}^{(k)}, \ldots, a_{s_{n} n}^{(k)} \in G$, $\alpha_{1 n}^{(k)}+\cdots+\alpha_{s_{n} n}^{(k)}=1, s_{n} \in \mathbb{N} ; c_{n}^{(k)} \in G$. It is a routine matter to see that $\prod_{i=1}^{n}\left(1+b_{i}^{p^{i-1}}-\right.$ $\left.b_{i+1}^{p^{i}}\right)$ is a canonical element with $\geq n$ members, and moreover because $G$ is separable as a subgroup of the separable $S(R G)$, there exists $u \in \mathbb{N}$ such that $b_{i}^{p^{i-1}} \notin G^{p^{u}}, b_{i}^{p^{i-1}} b_{j}^{\varepsilon p^{j-1}} \notin$ $G^{p^{u}}, \ldots, b_{i}^{p^{i-1}} b_{j}^{\varepsilon p^{j-1}} \cdots b_{l}^{\varepsilon p^{l-1}} \notin G^{p^{u}}$ (n members) for $1 \leq i \leq j \leq \cdots \leq l \leq n$ and $\varepsilon= \pm 1$. Furthermore it is a simple exercise to verify that the left hand-side of (o) contains a number $t$ of elements with nonzero coefficients which is strictly less than the number of the elements with nonzero coefficients in the right hand-side. This contradiction leads us to the fact that $\Phi_{n}$ is unconvergent in $S(R G) / G$. Finally we wish to apply the topological 
criterion of L. Kulikov $[8,6]$ to get that $G$ must be bounded, as claimed. The proof is finished.

An immediate consequence is the following due to N. Nachev (cf. [7] or [6] too).

Corollary 1. Let $G$ be a p-group. Then $S(R G)$ is closed if and only if $G$ bounded.

2. Quasi-closed $S(R G)$ for $N(R)=0$

Recall that as usual $N(R)$ is the nilradical in $R$. The main theorem here, however, is the following

Theorem 2. Presume $N(R)=0$. Then $S(R G)$ is quasi-closed if and only if $G_{p}$ is bounded.

Remark. The same theorem was proved by us in [7] but when $R$ is a field by making use of a purely algebraic technique.

In particular, we directly yield (cf. $[3,7]$ ).

Corollary 2. Let $N(R)=0$. Then $S(R G)$ is closed if and only if $G_{p}$ is bounded.

Now we are ready to obtain

Proof of Theorem 2. If $G_{p}$ is bounded, then certainly so is $S(R G)$ (see $[3,5]$ ), whence it is quasi-closed.

Now we treat the more difficult converse claim. Really, if $G_{p}$ is unbounded, the Main Lemma and second proposition imply $S(R G) / G_{p}$ is closed. Hence the same sequence $\left(\varphi_{n}\right)_{n=1}^{\infty}$ from the $p$-primary case but when $B$ is basic in $G_{p}$, along with the sequence $\Phi_{n}=\varphi_{n} G_{p} \in S(R G) / G_{p}(n \in \mathbb{N})$, yield the fact immediately. This completes the proof after all.

Remark. By what we have shown above and more specially according to our observation that the quasi-completeness preserves between $S(R G)$ and $S^{p^{s}}(R G)=S\left(R^{p^{s}} G^{p^{s}}\right)$, we derive that we can formulate: if $N\left(R^{p^{s}}\right)=0$ for any $s \in \mathbb{N}$, then $S(R G)$ is quasi-closed if and only if $G_{p}$ is bounded.

We close the main three cases of quasi completeness with

3. Quasi-closed $S(R G)$ for $N(R) \neq 0$

First and foremost we obtain the major extension of [6, Theorem], namely

Theorem 3. Suppose $N\left(R^{p^{w}}\right) \neq 0$ and $G$ is an abelian group. Then $S(R G)$ is closed if and only if $G$ is bounded p-primary.

Proof. Observe that the same sequence $f_{n}=\prod_{i=1}^{n}\left(1+r\left(1-g^{p^{i}}\right)\right)$ as in [6], but when $0 \neq r \in N\left(R^{p^{w}}\right)$ with $r^{2}=0 ; g \in G$ with $\operatorname{order}(g)=\infty$, together with the same conclusions as in [6, p.319, Case 1], yield that $G$ is torsion.

But then $T=\coprod_{q \neq p} G_{q}=1$ as a $p$-divisible group, since our proposition proved above means that $G$ is $p$-separable, whence $p$-reduced. Therefore $G$ is $p$-torsion and so Corollary 1 is applicable. The proof is completed. 
Of some interest is the situation when $N\left(R^{p^{w}}\right)=0$ and $N(R) \neq 0$ (or owing to the Remark of [6] or to this formulated above, $N\left(R^{p^{n}}\right) \neq 0$ for each $n \in \mathbb{N}$ is interesting), but the problem is still left-open to the moment and seems to be difficult.

The main theorem that determines this section, however, states thus.

Theorem 4. Suppose $R$ is such a ring that $N\left(R^{p^{w}}\right) \neq 0$ and either (*) $G / G_{p}$ is p-divisible or $(* *) G / G_{p}$ is decomposable.

Then $S(R G)$ is quasi-closed if and only if $G$ is a bounded p-group.

Proof. It is a development of our idea in [6]. The condition that $G$ is bounded $p$-torsion implies the same for $S(R G)$ [4], hence it is quasi-closed [8].

Now, for the converse, choose $S(R G)$ quasi-closed. If $G_{p}$ is unbounded, then as above $S(R G) / G_{p}$ must be closed and thus as in the proofs of Theorems 2 or 3 we will obtain that $G_{p}$ is bounded. Consequently $G=G_{p} \times M[8]$ for some $M \leq G$. Now, if (*) is fulfilled, then as above $M=1$ (cf. [6]) and so $G=G_{p}$. Furthermore $G$ is bounded $p$-torsion. Further, if $(* *)$ holds, then owing to [4] we claim that $S(R M)$ is a direct factor of $S(R G)$ and thus the first proposition implies that $S(R M)$ is quasi-closed. The hypothesis on $M \neq 1$ leads us to $M=A \times B$, where both $1 \neq A, B \neq M$. Applying [4] and the second proposition we derive $S(R M) / S(R A) \cong S((R A) B)$ is closed, because $S(R A)$ is unbounded pure (otherwise $A$ must be $p$-torsion [5], which is wrong). That is why by virtue of Theorem 3 we deduce $B$ is $p$-primary since $(R A)^{p^{w}}=R^{p^{w}} A^{p^{w}}$ possesses nilpotents. The contrary $B=B_{p}=1$ ensures that $M=1$. Thus $G=G_{p}$ once again. This means $G$ is bounded $p$-primary. The proof is completed after all.

The author feels that the answer of the following question would be of some interest and importance to the theory of the commutative group algebras.

Problem. Presume that $G$ is a torsion-free indecomposable group. Whether $S(R G)$ is quasi-closed if and only if $G=1$, provided that $N\left(R^{p^{w}}\right) \neq 0$ ? The positive solution of the last problem guarantees that Theorem 4 will be true in a general form, i.e. for arbitrary $G$ and $N\left(R^{p^{w}}\right) \neq 0$. But besides when $N\left(R^{p^{w}}\right)=0$ with $N(R) \neq 0$ (or more precise owing to the Remark, for $N\left(R^{p^{n}}\right) \neq 0$ with every $n \in \mathbb{N}$ ), the situation is left-open yet, too.

Correction. The first line of Case 2 from our article [6] should be read thus: Then $G=\coprod_{p} G_{p}=\coprod_{q \neq p} G_{q} \times G_{p}$ ( $q$ is a prime), where $T=\coprod_{q \neq p} G_{q}$ is a $p$-divisible group, i.e. $T^{p}=T$, because $G_{q}^{p}=G_{q}$ for all $q \neq p ;(q, p)=1$.

\section{Acknowledgement}

The author would like to express his warm gratitude to Professor Kar-Ping Shum for the precise editorial advice. 


\section{References}

[1] K. M. Benabdallah, B. J. Eisenstadt, J. M. Irwin and E. W. Poluianov, The structure of large subgroups of primary abelian groups, Acta Math. Hung. 21(1970), 421-435.

$[2]$ D. O. Cutler, Quasi-isomorphism for infinite abelian p-groups, Pac. J. Math. 16(1966), $25-45$.

[3] P. Danchev, Sylow p-subgroups of commutative group algebras, Compt. Rend. Acad. Bulg. Sci. 46(1993), 13-14.

[4] P. Danchev, Normed unit groups and direct factor problem for commutative modular group algebras, Math. Balkanica 10(1996), 161-173.

[5] P. Danchev, Topologically pure and basic subgroups in commutative group rings, Compt. Rend. Acad. Bulg. Sci. 48(1995), 7-10.

[6] P. Danchev, Torsion completeness of Sylow p-groups in modular group rings, Acta Math. Hung. 75(1997), 317-322.

[7] P. Danchev, Quasi-completeness in commutative modular group algebras, Ricerche Mat. 51(2002).

[8] L. Fuchs, Infinite Abelian Groups, vols. I and II, Mir, Moscow, 1974 and 1977. (Russian)

[9] P. Hill and C. Megibben, Quasi-closed primary groups, Acta Math. Hung. 16(1965), 271274.

[10] G. Karpilovsky, Unit Groups of Group Rings, North-Holland, Amsterdam, 1989.

Department of Mathematics, Plovdiv State University, 4000 Plovdiv, Bulgaria. 